\title{
Patient Registries: An Underused Resource for Medicines Evaluation
}

\section{Operational proposals for increasing the use of patient registries in regulatory assessments}

\author{
Patricia McGettigan ${ }^{1}$ (1) . Carla Alonso Olmo ${ }^{2} \cdot$ Kelly Plueschke $^{2} \cdot$ Mireia Castillon $^{2} \cdot$ Daniel Nogueras Zondag $^{2}$. \\ Priya Bahri ${ }^{2} \cdot$ Xavier Kurz ${ }^{2}$ Peter G. M. Mol ${ }^{3,4}$
}

Published online: 13 July 2019

(c) The Author(s) 2019

\begin{abstract}
Introduction Patient registries, 'organised systems that use observational methods to collect uniform data on a population defined by a particular disease, condition, or exposure, and that is followed over time', are potentially valuable sources of data for supporting regulatory decision-making, especially for products to treat rare diseases. Nevertheless, patient registries are greatly underused in regulatory assessments. Reasons include heterogeneity in registry design and in the data collected, even across registries for the same disease, as well as unreliable data quality and data sharing impediments. The Patient Registries Initiative was established by the European Medicines Agency in 2015 to support registries in collecting data suitable to contribute to regulatory assessments, especially post-authorisation safety and effectiveness studies.

Methods We conducted a qualitative synthesis of the published observations and recommendations from an initiativeled multi-stakeholder consultation and four disease-specific patient registry workshops. We identified the primary factors facilitating the use of registry data in regulatory assessments. We generated proposals on operational measures needed from stakeholders including registry holders, patients, healthcare professionals, regulators, marketing authorisation applicants and holders, and health technology assessment bodies for implementing these.

Results Ten factors were identified as facilitating registry use for supporting regulatory assessments of medicinal products. Proposals on operational measures needed for implementation were categorised according to three themes: (1) nature of the data collected and registry quality assurance processes; (2) registry governance, informed consent, data protection and sharing; and (3) stakeholder communication and planning of benefit-risk assessments.

Conclusions These are the first explicit proposals, from a regulatory perspective, on operational methods for increasing the use of patient registries in medicines regulation. They apply to registry holders, patients, regulators, marketing authorisation holders/applicants and healthcare stakeholders broadly, and their implementation would greatly facilitate the use of these valuable data sources in regulatory decision-making.
\end{abstract}

Electronic supplementary material The online version of this article (https://doi.org/10.1007/s40264-019-00848-9) contains supplementary material, which is available to authorized users.

Patricia McGettigan

p.mcgettigan@qmul.ac.uk

1 William Harvey Research Institute, Queen Mary University of London, Charterhouse Square, London EC1M 6BQ, United Kingdom

2 Pharmacovigilance and Epidemiology Department, European Medicines Agency, Amsterdam, Netherlands

3 Department of Clinical Pharmacy and Pharmacology, University Medical Centre Groningen, University of Groningen, Groningen, The Netherlands

4 Dutch Medicines Evaluation Board, Utrecht, The Netherlands

\section{Introduction}

Health-related real world data provide crucial support for regulatory decision-making, especially in post-authorisation assessments of medicinal products [1]. There are multiple sources including patient (disease) registries, electronic health records, insurance claims databases, health surveys, and prescription dispensing databases [2]. Patient registries, 'organised systems that use observational methods to collect uniform data on a population defined by a particular disease, condition, or exposure, and that is followed over time', are a potentially rich source of data, especially for evaluating the course of rare diseases and effects of new treatments [3-5]. Despite this, they are greatly underused in regulatory 


\section{Key Points}

Patient registries are potentially valuable sources of data for supporting regulatory decision-making on medicines, but they are greatly underused owing to heterogeneity in registry design, the data collected and its quality, as well as to data sharing impediments.

The European Medicines Agency's Patient Registries Initiative aims to support registries in collecting data suitable to contribute to regulatory assessments, especially post-authorisation safety and effectiveness studies.

We have generated operational proposals on patient registry data, quality assurance processes, governance and stakeholder communication that will help to increase the use of these valuable resources in regulatory benefit-risk assessments of medicines.

assessments of medicines. There are many reasons, including heterogeneity in registry design within individual disease areas, unreliable data quality and data sharing barriers, all amplified by limited national and international collaboration $[5,6]$.

\subsection{Use of Patient Registries for Supporting Regulatory Assessments}

In pivotal studies supporting marketing authorisation of medicinal products, randomised controlled trial (RCT) data are preferred by regulators. However, in situations where RCT data are limited or where RCTs are not ethical or are not feasible, as with many rare diseases, patient registry data may provide crucial support for regulatory decision-making. For example, in the case of haemophilia, the updated guideline on Factor VIII products removes the obligation to perform clinical trials in previously untreated patients but requires post-authorisation studies based on a set of core data elements to be collected in patient registries [7]. For products granted conditional marketing approval, registry studies may provide post-authorisation data to fulfil regulator-imposed specific obligations to confirm safety and/ or effectiveness, as is the case with the recently authorised chimeric antigen receptor (CAR) T-cell products, tiagenlecleucel and axicabtagene ciloleucel $[8,9]$. Some registries may be of particular value in terms of the patient population size and representativeness, the duration of follow-up data for treatment-exposed patients and availability of information not collected in other real world repositories.

Notwithstanding such potential, the under-use of patient registries in the regulatory context is striking $[10,11]$. Of
335 products recommended for approval between 2005 and 2013 by the main scientific committee of the European Medicines Agency (EMA), the Committee for Medicinal Products for Human Use (CHMP), 31 registries were requested to fulfil a condition of the marketing authorisation, but by December 2017, just ten had been completed [11]. Potentially, registries could provide data permitting comparisons of outcomes from different treatments across different countries and healthcare settings over time as well as assessment of the impact of measures taken to minimise risks of medicinal products $[12,13]$. Their limitations for such assessments are well-described, but a description of the features needed to encourage increased use is lacking [4, 12-14]. Our aim with this work was to provide proposals, from a regulatory perspective, on how registry stakeholders could fill this gap.

\section{Methods}

We conducted a qualitative synthesis of the observations and recommendations published in five reports arising from a multi-stakeholder consultation and four disease-specific workshops conducted as part of EMA's Patient Registries Initiative.

From the consultation report, we identified the primary factors considered by stakeholders as facilitating the use of registry data for supporting medicines regulation.

From each of the four disease-specific workshop reports, we abstracted the participant observations and recommendations on utilising patient registry data in regulatory assessments along with the measures needed from stakeholders in order to implement them. We then generated operational proposals, applicable to patient registries and stakeholder groups broadly, for implementing these measures.

\section{Patient Registries Initiative}

In 2015, EMA established a Patient Registries Initiative to support a systematic and standardised approach for registry contribution to medicines assessment, especially for postauthorisation safety studies (PASS) and post-authorisation effectiveness studies (PAES) [6]. The initiative aims to create a registry framework with collaboration between registry coordinators, including healthcare professionals' and patients' associations, academic institutions and national agencies responsible for overseeing healthcare services, and potential users of registry data, such as medicines regulators, reimbursement bodies, and pharmaceutical companies. Key elements of its strategy include facilitating the use of existing patient registries within the current legal and regulatory framework for medicinal products and providing methodological support for the establishment of new registries [15]. 


\section{Multi-Stakeholder Consultation and Disease-Specific Registry Workshops}

At a consultation in October 2016, 122 expert stakeholders, including registry holders, patients, healthcare professionals, regulators, marketing authorisation holders and applicants (MAHs/MAAs), and health technology assessment (HTA) and reimbursement bodies, and European Commission representatives shared their views on barriers to and facilitators of registry use and on optimising the use of registries for regulatory assessments [16]. The discussions provided the groundwork for four disease-specific registry workshops held during 2017 and 2018 that explored the use of registry data for supporting regulatory assessments in four areas of active product development where new products had recently been approved or were undergoing assessment (Appendix Table 1, see the electronic supplementary material): cystic fibrosis [17], multiple sclerosis [18], CAR T-cell therapies [19], and haemophilia therapies [20].

The four workshops together included 266 participants representing all of the stakeholder groups in each case. The individual workshop reports providing participants' observations and recommendations, along with the report of the multi-stakeholder consultation, are published on the EMA patient registries webpage [6]. The objectives and methods of the individual patient registry workshops are described in Appendix Table 2 (see the electronic supplementary material). This work did not require ethics approval.

\section{Results}

\subsection{Factors Supporting the Use of Registries}

Synthesis of the participant observations from the multistakeholder consultation report generated a list of factors that facilitated the use of patient registries for regulatory assessments. They included the use of common core data sets, common coding terminologies, complete data collection, especially on medications, facility for data access and sharing, data linkage capacity, quality assurance processes and governance, early consideration of registries in the regulatory process, stakeholder communication, registry sustainability and the availability of a registry framework. Their value in facilitating registry use is described in Table 1 . In each case, absence or incompleteness greatly impeded registry use.

\subsection{Proposals on Operational Measures to Increase Registry Use in Regulatory Assessments}

Three themes, generalisable to patient registries broadly, emerged from the published observations and recommendations made by the participants in each of the four disease-specific patient registry workshops:

1. Nature of the data collected and registry quality assurance processes

2. Registry governance, informed consent, data protection and sharing

3. Stakeholder communication and planning of benefit-risk assessments.

\subsubsection{Nature of the Data and Registry Quality Assurance Processes}

The need for registries in a given disease area to collect core common data elements, commonly defined, was acknowledged by all stakeholders as essential for ensuring that data from multiple registries in a given disease area could be combined to enhance both the generalisability and the power of studies that could be conducted using the data (Table 2, Box 1). EMA scientific advice may assist in clarifying the suitability of individual registries for defined purposes [23]. Knowledge of data quality is fundamental for regulatory assessments. Quality may be judged according to three components: consistency, accuracy, and completeness. Table 2 defines each quality component, summarises potential indicators of quality that could be applied in registries, and describes the systems or solutions needed to facilitate these in operational terms.

\subsubsection{Registry Governance, Informed Consent, Data Protection and Sharing}

Proposals are summarised in Table 3 for measures needed on registry governance, informed consents, and data sharing and protection in order to ensure that data are accessible for regulatory assessments and may be shared in the context of the applicable legal and governance frameworks.

Examples of recommendations from the individual workshop reports are quoted in Box 2. Registry sustainability measures were not a focus of the disease-specific workshop discussions given the regulatory context, but were acknowledged by all stakeholders as crucial for registry stability and development.

\subsubsection{Communication with Stakeholders}

Acknowledged areas of improvements needed to support the use of registry data in regulatory assessments include communication between stakeholders early in the marketing authorisation process in order to plan for post-authorisation studies (Fig. 1, Box 3). The studies are needed so that marketing authorisation, if granted, may be followed-up with timely evidence on the benefit-risk balance of new products 
Table 1 Factors facilitating registry use for supporting regulatory assessments

\begin{tabular}{|c|c|}
\hline Factor & Value in supporting registry use for regulatory assessments \\
\hline Use of common core data sets & $\begin{array}{l}\text { Collecting a common core set of data items with agreed definitions and data dictionaries increases the } \\
\text { capacity to combine or pool data across patients or registries for regulatory assessments. Ideally, data } \\
\text { items match regulatory needs. Capacity to collect additional data elements, even for a limited period, } \\
\text { may be beneficial. }\end{array}$ \\
\hline Common data coding terminologies & $\begin{array}{l}\text { The availability of coding terminologies such as the Medical Dictionary for Regulatory Activities } \\
\left(\text { MedDRA }{ }^{\circledR} \text { ) that could be used by all registries helps in facilitating the conduct of studies using data }\right. \\
\text { from multiple registries [21]. }\end{array}$ \\
\hline Complete information collection & $\begin{array}{l}\text { Complete information on critical disease variables is necessary. Medication information is often limited; } \\
\text { primary disorder medication information is essential and should include the start and stop (where } \\
\text { applicable) dates. Most registries do not record other medications, but some information is desirable. } \\
\text { PROs are of increasing interest to stakeholders, but are not collected in most registries. }\end{array}$ \\
\hline Data access and sharing & $\begin{array}{l}\text { Clear consent specifications on data use facilitate sharing of registry data with third parties including } \\
\text { regulators and MAAs/MAHs. Data sharing and access are further determined by relevant national and } \\
\text { European data protection legislation. }\end{array}$ \\
\hline Data linkage capacity & $\begin{array}{l}\text { Linkages to external databases, for example, prescription dispensing, employment, or death registries } \\
\text { add to the value of registry data, but linkages may be variable across member state. }\end{array}$ \\
\hline $\begin{array}{l}\text { Registry reporting, and quality assur- } \\
\text { ance processes and governance }\end{array}$ & $\begin{array}{l}\text { Most registries have processes in place for annual reporting and for quality assurance including source } \\
\text { data verification. While these are heterogeneous currently, they represent good baselines for further } \\
\text { development in individual registries. }\end{array}$ \\
\hline Timeliness of consideration & $\begin{array}{l}\text { Consideration of registry data in the authorisation process generally occurs when risk management plans } \\
\text { and post-authorisation data needs are being discussed. Planning early in the authorisation process for } \\
\text { registry use facilitates data access by reducing timelines for data upload from treating centres and for } \\
\text { registry quality assurance processes. }\end{array}$ \\
\hline Direct communication & $\begin{array}{l}\text { To best fulfil regulator-requested or regulator-imposed studies, regulators, MAAs/MAHs and registry } \\
\text { holders need to communicate directly. }\end{array}$ \\
\hline Sustainability & $\begin{array}{l}\text { Registry funding and support may be limited, causing difficulties in maintaining database systems, reli- } \\
\text { able quality assurance processes, and staff training. Data entry is often done on a voluntary basis and } \\
\text { manually by clinical staff either directly or by importing information from electronic health records. } \\
\text { Registry sustainability is crucial for long-term development. }\end{array}$ \\
\hline Availability of a regulatory framework & $\begin{array}{l}\text { EMA guidelines and procedures for PASS and PAES provide a structure for stakeholder dialogue on } \\
\text { registry use [22]. EMA scientific advice can support CHMP qualification opinions or advice on the } \\
\text { suitability of a registry for undertaking pharmacoepidemiological studies [23]. }\end{array}$ \\
\hline
\end{tabular}

CHMP Committee for Medicinal Products for Human Use, EMA European Medicines Agency, MAAs/MAHs marketing authorisation applicants/ holders, PAES post-authorisation efficacy studies, PASS post-authorisation safety studies, PROs patient reported outcomes

for patient and public health, most especially for products with specific obligations where delays in study completion are common [25-27]. Opportunities arise early in the marketing authorisation process to pro-actively identify likely data needs, especially for post-authorisation studies. These opportunities are illustrated in Fig. 1. Where registry data could potentially contribute, there should be three-way contact involving regulators, MAAs/MAHs and registry holders to explore data availability.

\section{Discussion}

\subsection{Priorities for Implementation of Proposals}

Our proposals have been synthesised from the published reports of a consultative discussion and four disease-specific workshops that together included almost 400 specialist stakeholders with patient registry expertise. In doing so, we have leveraged the deep knowledge of participants in each disease area to generate proposals that apply to registries broadly. Implementation of the proposals by registry stakeholders collectively would help to establish a harmonised patient registry environment within many other individual disease areas, thereby increasing the suitability of registry data for regulatory assessments of related medicinal products.

From a regulatory perspective, the priorities for the development of a European Union-wide framework on patient registries are:

- Availability of core common data sets, specific for individual disease areas, with commonly defined data elements across registry networks

- Registry operational procedures for MAAs/MAHs and regulators to access data in accordance with national regulation and European General Data Protection Regulation [20]

- Transparent quality assurance processes in registries. 
Table 2 Proposals on data elements and data quality attributes necessary in patient registries and on the operational measures required for implementation

\begin{tabular}{|c|c|c|}
\hline Topic & Proposals & Operational measures required \\
\hline \multirow[t]{3}{*}{ Core common data elements } & $\begin{array}{l}\text { Core common data elements to be collected by all } \\
\text { contributing registries in a specific disease area }\end{array}$ & $\begin{array}{l}\text { Agree on the core common data elements to be included } \\
\text { in specific disease area registries, including the associ- } \\
\text { ated definitions and data dictionaries }\end{array}$ \\
\hline & Harmonise data element definitions across registries & $\begin{array}{l}\text { Provide data element definition information or source to } \\
\text { stakeholders }\end{array}$ \\
\hline & $\begin{array}{l}\text { Agree on core PROs that could feasibly be collected } \\
\text { systematically }\end{array}$ & $\begin{array}{l}\text { All stakeholders to collaborate on defining PROs (appro- } \\
\text { priate as necessary for patient age, capacity, language, } \\
\text { and for caregivers) }\end{array}$ \\
\hline Data quality & $\begin{array}{l}\text { Indicators on data consistency, accuracy and com- } \\
\text { pleteness to be implemented and reported }\end{array}$ & $\begin{array}{l}\text { Registries to publish at agreed intervals reports or audits } \\
\text { of data quality }\end{array}$ \\
\hline Quality components & Indicators of quality & Operational measures required \\
\hline $\begin{array}{l}\text { Consistency: } \\
\text { Uniformity of the data over } \\
\text { time (e.g. laboratory data } \\
\text { routinely entered) }\end{array}$ & $\begin{array}{l}\text { Proportion of data fields changed over time } \\
\text { Proportion of fields missing over time }\end{array}$ & $\begin{array}{l}\text { Audits and centre level data checks } \\
\text { Standard terminology and coding } \\
\text { Standard operating procedures } \\
\text { Registry data entry dashboard }\end{array}$ \\
\hline $\begin{array}{l}\text { Accuracy: } \\
\text { Accuracy of data entry-no } \\
\text { errors, contradictions or } \\
\text { impossibilities in the data } \\
\text { Absence of duplicates }\end{array}$ & $\begin{array}{l}\text { Change in value of data filed by } x \% \text { creates alerts } \\
\text { Variability of data values across common fields }\end{array}$ & $\begin{array}{l}\text { Drop down menus, alerts, text prompts } \\
\text { Validate registry data sample (e.g. 10\%) against source } \\
\quad \text { data } \\
\text { Software checks } \\
\text { Staff training } \\
\text { Help screens/desks } \\
\text { Funding for data managers }\end{array}$ \\
\hline $\begin{array}{l}\text { Completeness: } \\
\text { Proportion of data missing } \\
\text { Absence of core variables }\end{array}$ & $\begin{array}{l}\text { Agreed } \% \text { of fields completed in audit procedures } \\
\text { (e.g. }>90 \%) \\
\text { Proportion of patients lost to follow-up/attrition rates } \\
\text { Minimum agreed core common data elements reported } \\
\text { All treated patients reported, not selected patients only }\end{array}$ & $\begin{array}{l}\text { Audits } \\
\text { Mandatory fields } \\
\text { Agreement on entry of 'not done' or 'null' values } \\
\text { Engagement with patients and HCPs } \\
\text { Agreed list of data elements and definitions } \\
\text { Cross-check patient numbers with numbers of products } \\
\text { used at treating centres (applicable for some advanced } \\
\text { therapies) }\end{array}$ \\
\hline
\end{tabular}

HCPs healthcare professionals, PROs patient reported outcomes

Box 1 Nature of the data collected and registry quality assurance processes

Workshop participants' recommendations included:

'Agree on standards for data quality indicators, terminologies/coding and reporting requirements to apply to national registries and to the ECFSPR' (European Cystic Fibrosis Society Patient Registry) [17].

'Agreement on the data elements to be collected in MS (multiple sclerosis) registries would facilitate treatment evaluations and comparisons of safety and effectiveness outcomes between different MS populations and across multiple countries' [18].

'Established quality standards should be in place and adequate for all registry studies; a dedicated data control and follow-up system should be introduced only for very specific studies or where the existing system is not [yet] adequate' [19].

'Definitions for the data elements required by the FVIII Guideline need to be agreed and applied across treating centres and registries; the associated data dictionaries need to be established and maintained' [20].

Therefore, in addressing the factors described in Table 1, the proposals on data elements, quality assurance, governance, patient consents, and data protection summarised in Tables 2 and 3, together with communication and planning early in the authorisation process, are critical if the potential of patient registry data for regulatory assessments of medicinal products is to be realised.

\subsection{EMA Actions to Increase Registry Use}

EMA has provided scientific advice to support CHMP qualification opinions on two patient registries regarding their suitability for supporting regulatory assessments of medicines, the European Cystic Fibrosis Society Patient Registry and the cellular therapy module of the European Blood and 
Table 3 Proposals on measures required for registry governance, informed consent, data protection and sharing

\begin{tabular}{ll}
\hline Topic & Proposals for measures needed from stakeholders \\
\hline Registry governance & Regulators and/or MAAs/MAHs to identify early in the authorisation process whether a potentially relevant \\
& registry exists and identify data elements needed, especially for post-authorisation assessments likely to be \\
& requested or imposed, and to agree on a common study protocol. \\
Regulators and MAAs/MAHs to be aware of the data elements that can feasibly be collected systematically by \\
relevant registries and to inform registries on their data needs. \\
Registry holders to establish a centralised data application process (with a standard template) for stakeholders \\
to request and obtain data. \\
Communicate to patients and the public the benefits and uses of patient registry data and the value of high \\
levels of patient inclusion in registries. \\
Registry holders to ensure clinical/treating centres confirm that registry patients have provided consent and \\
review whether current patient consent is broad enough for possible future situations taking into account \\
European GDPR [24]. \\
Registry holders to develop a policy on data analysis and sharing summary, pseudo-anonymised, and indi- \\
vidual patient data that aligns with national regulation and European GDPR. \\
Data sharing and data protection consent
\end{tabular}

GDPR General Data Protection Regulation, $M A A / M A H$ marketing authorisation applicant/holder

Box 2 Registry governance, informed consent, data protection and sharing

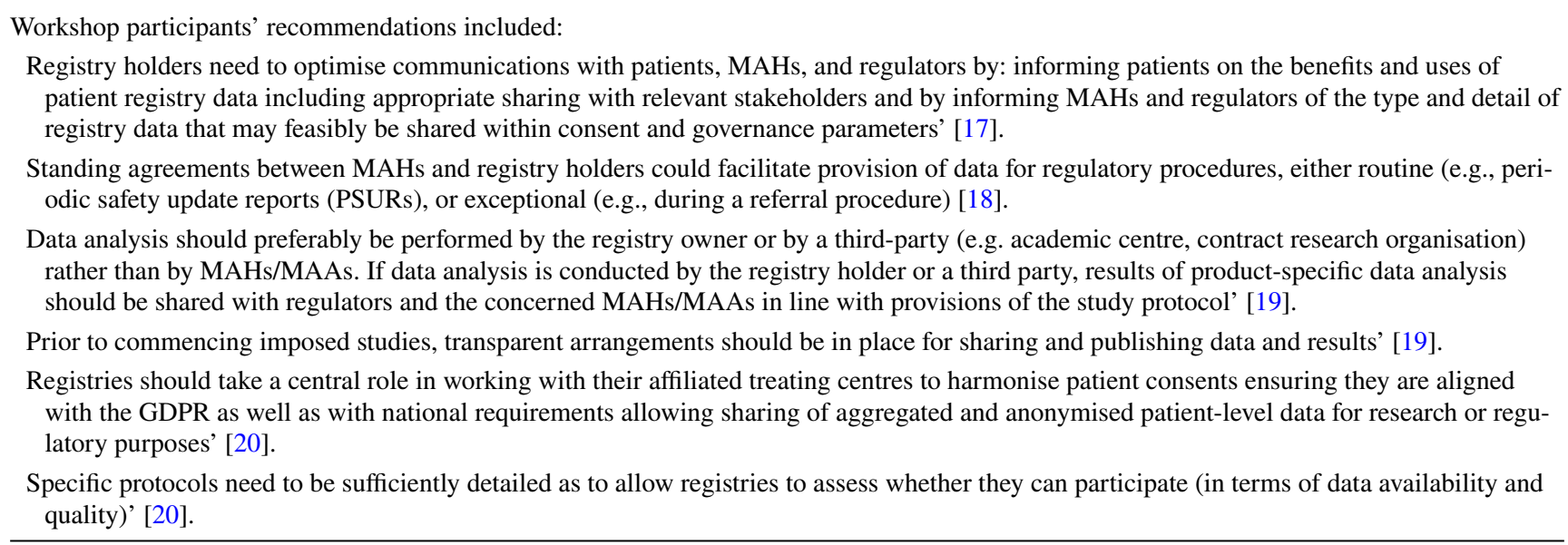

Marrow Transplant registry [19]. In both cases, the opinions describe contexts of use for which the registry data are considered suitable by CHMP for undertaking pharmacoepidemiology studies. The possibility for registries to obtain scientific advice to support a qualification opinion may go some considerable way in assuring stakeholders that registries so qualified are satisfactory for regulatory studies.

Internally, EMA has instituted measures to identify products during pre-submission stages of authorisation processes (pre-submission meetings, scientific advice and priority medicines [PRIME] discussions [28]) (Figure 1) where registry or other real world data may be needed for post-authorisation follow-up if marketing authorisation is granted. This action anticipates and permits pro-active planning for post-authorisation assessments and reflects the regulatory policy of benefit-risk assessment throughout the product lifecycle. To assist stakeholders in identifying potentially relevant registries, a publicly available inventory of patient registries is hosted on the European Network of Centres for Pharmacoepidemiology and Pharmacovigilance (ENCePP) resources database [29].

EMA should not duplicate other initiatives aiming to enhance registry use in healthcare. Therefore, its patient registries strategy aligns with the European Commission policy framework on rare diseases as well as with priorities of the European Research Networks for rare diseases, the Horizon 2020 programme and Joint Action initiatives such as the European Network for Health Technology Assessment (EUnetHTA), and takes into account national endeavours such as those underway in the Netherlands and Sweden [30-35]. The European Platform on Rare Diseases Registration (EU RD Platform) has developed a 'Set of common data 


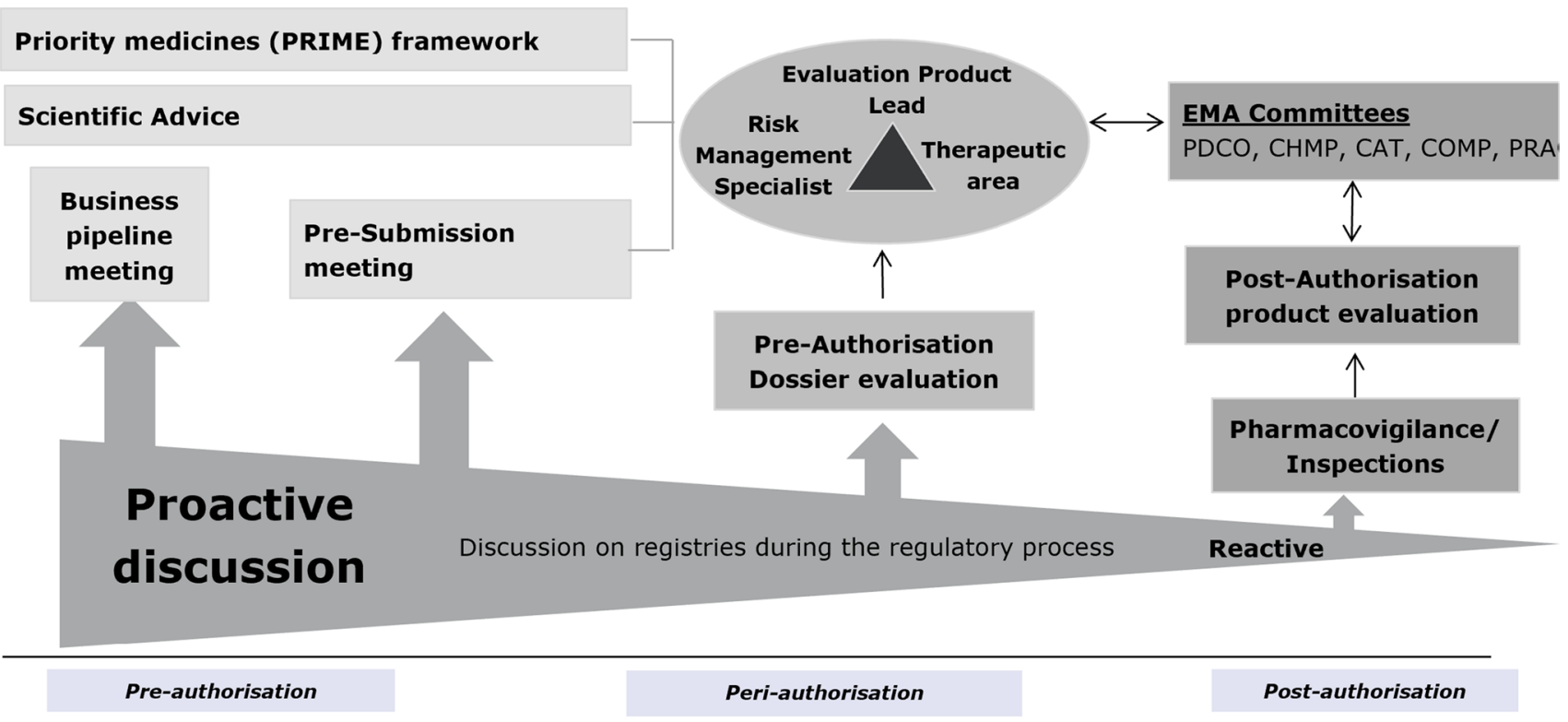

Fig. 1 Opportunities during the regulatory cycle to identify where registry data may be needed for post-authorisation follow-up. Source: Clin Pharmacol Ther 2019 https://doi.org/10.1002/cpt.1414

Box 3 Stakeholder communication and planning of benefit-risk assessments

Workshop participants' recommendations included:

Communicate the value of registries, their limitations, and the importance of consistent data quality to all participating healthcare professionals and to those using the data including MAHs, regulators, HTA and reimbursement bodies' [17].

MAHs, regulators and registry holders, plus other stakeholders where relevant (for example, reimbursement bodies), should engage in discussions early during the regulatory processes for approval of new treatments to consider data needs and scientific / study protocols and to understand the range and nature of data that registries could provide, especially for post-authorisation studies' [18].

MAHs / MAAs need to 'commence planning for post-authorisation data collection early in product development' and 'develop a preliminary study protocol and explore with the registry holder/s and regulators if the registry could fulfil the data needs, for example, through a scientific advice procedure' [19].

MAAs must '[i]nitiate discussions with registries and regulators before or at an early stage of a marketing authorisation application on the relevance and adequacy of one or several existing disease registries for the long-term monitoring of their specific product' [20].

elements for RD Registration' [36]. It is aimed at the European Reference Network's existing registries and registries under development; other rare disease registries at national, regional, and local levels in EU Member States; researchers and patient organisations.

From a regulatory perspective, the ultimate requirement of patient registries is that they permit the conduct of highquality studies that evaluate the safety and effectiveness of medicines. Recognising the benefits and challenges inherent in using observational data for medicines assessments, in 2019, EMA will publish methodological and operational advice on handling registry data in post-authorisation studies, taking into account responses to its open consultation on a preliminary discussion paper [6]. Application of the advice will be underpinned by clear understanding of the differences between a registry and a registry study (Table 4).

\section{Conclusions}

This is the first time that the factors necessary for patient registry data to adequately support regulatory assessments, together with operational proposals required for their implementation, have been set out explicitly from a regulatory perspective. In explaining what is needed, taking account of the current legal and regulatory framework for medicinal products, the proposals empower stakeholders seeking to capitalise on the potential of patient registries broadly to 
Table 4 Methodological aspects: distinguishing a registry from a registry study

\begin{tabular}{|c|c|c|}
\hline Characteristic & Registry & Registry study \\
\hline Nature & Data collection system & Investigation of a research question or hypothesis \\
\hline Timelines & Long-term, open-ended & $\begin{array}{l}\text { Defined by the study objective and described in the study } \\
\text { protocol }\end{array}$ \\
\hline Patient enrolment & $\begin{array}{l}\text { Exhaustive within the boundaries of the purpose of the } \\
\text { registry (e.g. all patients diagnosed with a disease in a } \\
\text { hospital, region or country) }\end{array}$ & $\begin{array}{l}\text { Defined by research objective and described in the study } \\
\text { protocol-it may be a subset of the registry population }\end{array}$ \\
\hline Data collection & $\begin{array}{l}\text { Wide range of data may be collected depending on the } \\
\text { purpose of the registry }\end{array}$ & $\begin{array}{l}\text { Restricted to what is needed by the research question } \\
\text { including data on potential confounders and effect } \\
\text { modifiers - additional data collection may be required }\end{array}$ \\
\hline Analysis plan & $\begin{array}{l}\text { Routine periodical data analysis; additional ad-hoc } \\
\text { analyses }\end{array}$ & $\begin{array}{l}\text { Statistical analysis plan separate from the study protocol } \\
\text { in line with the objectives }\end{array}$ \\
\hline $\begin{array}{l}\text { Collection and reporting } \\
\text { of suspected adverse } \\
\text { reactions }\end{array}$ & $\begin{array}{l}\text { National requirements as regards the management of } \\
\text { safety data apply. Any active data collection with } \\
\text { involvement of a MAH must follow the regulatory } \\
\text { framework for PASS }\end{array}$ & $\begin{array}{l}\text { National requirements may apply. Regulatory require- } \\
\text { ments to MAHs differ between studies with primary or } \\
\text { secondary data collection }\end{array}$ \\
\hline Data quality control & Applied routinely to all data and processes & Additional quality assurance may be needed \\
\hline Regulatory status & Non-interventional & Non-interventional or interventional \\
\hline
\end{tabular}

$M A H$ marketing authorisation holder, $P A S S$ post-authorisation safety study

support and contribute to regulatory decision-making on medicines.

Actions by all stakeholders, registry owners as well as MAAs/MAHs, regulators, patients/their representatives, healthcare professionals, and HTA and medicines reimbursement bodies, are required to implement the proposals and thereby consolidate registry value in patient and public health.

Acknowledgements We acknowledge the support of EMA's Cross Committee Task Force on Patient Registries. We thank Filipa Da Mota for commencing the Inventory of Registries and for her support, along with Valerie Muldoon, of the stakeholder consultation and patient registry workshops. We acknowledge the generous input of expertise and time by the multiple stakeholders who contributed to the consultation, the disease-specific workshops, and the creation of the five published reports arising from these endeavours.

\section{Compliance with Ethical Standards}

Funding No sources of funding were used to assist in the preparation of this study.

Conflict of interest The authors, Patricia McGettigan, Carla Alonso Olmo, Kelly Plueschke, Mireia Castillon, Daniel Nogueras Zondag, Priya Bahri, Xavier Kurz and Peter Mol, have no conflicts of interest that are directly relevant to the content of this study.

Patient consent Patient consent was not required for this work.

Disclaimer The views expressed in this article are those of the authors and may not be understood or quoted as reflecting the views of their respective institutions or of the European Medicines Agency or one of its committees or working parties.
Open Access This article is distributed under the terms of the Creative Commons Attribution-NonCommercial 4.0 International License (http://creativecommons.org/licenses/by-nc/4.0/), which permits any noncommercial use, distribution, and reproduction in any medium, provided you give appropriate credit to the original author(s) and the source, provide a link to the Creative Commons license, and indicate if changes were made.

\section{References}

1. Sherman RE, Anderson SA, Dal Pan GJ, Gray GW, Gross T, Hunter NL, et al. Real-world evidence-what is it and what can it tell us? N Engl J Med. 2016;375(23):2293-7.

2. Makady A, de Boer A, Hillege H, Klungel O, Goettsch W, on behalf of GetReal Work Package 1. What is real-world data? a review of definitions based on literature and stakeholder interviews. Value Health J Int Soc Pharmacoecon Outcomes Res. 2017;20(7):858-65.

3. Gliklich RE, Dreyer NA, eds. Registries for Evaluating Patient Outcomes: A User's Guide. Agency for Healthcare Research and Quality. 2007. https://effectivehealthcare.ahrq.gov/sites/default/ files/pdf/registries-guide_research.pdf. Accessed 30 June 2019.

4. Zaletel, M, Kralj, M, Magajne, M, Doupi, P. Methodological Guidelines and Recommendations for Efficient and Rational Governance of Patient Registries. PARENT. Vol. 25. 2015. https ://ec.europa.eu/health/sites/health/files/ehealth/docs/patient_regis tries_guidelines_en.pdf. Accessed 30 June 2019.

5. European Medicines Agency. Patient Registries. http://www.ema. europa.eu/ema/index.jsp?curl=pages/regulation/general/general_ content_000658.jsp\&mid=WC0b01ac0580961211. Accessed 30 June 2019.

6. Forrest CB, Bartek RJ, Rubinstein Y, Groft SC. The case for a global rare diseases registry. Lancet. 2011;377:1057-9.

7. European Medicines Agency. Guideline on the clinical investigation of recombinant and human plasma-derived factor VIII products [Draft]. https://www.ema.europa.eu/documents/scien 
tific-guideline/draft-guideline-clinical-investigation-recombinan t-4-human-plasma-derived-factor-viii-products_en.pdf. Accessed 30 June 2019.

8. European Medicines Agency. Kymriah. Summary of Product Characteristics. https://www.ema.europa.eu/documents/productinformation/kymriah-epar-product-information_en.pdf Accessed 25 Mar 2019.

9. European Medicines Agency. Yescarta. Summary of Product Characteristics. https://www.ema.europa.eu/documents/productinformation/yescarta-epar-product-information_en.pdf.

10. Jonker CJ, van den Berg HM, Kwa MSG, Hoes AW, Mol PGM. Registries supporting new drug applications. Pharmacoepidemiol Drug Saf. 2017;26(12):1451-7.

11. Pacurariu A, Plueschke K, Olmo CA, Kurz X. Imposed registries within the European post-marketing surveillance system: extended analysis and lessons learned for regulators. Pharmacoepidemiol Drug Saf. 2018;27(7):823-6. https://doi.org/10.1002/pds.4449.

12. Bessonova L, Volkova N, Higgins M, Bengtsson L, Tian S, Simard $\mathrm{C}$, et al. Data from the US and UK cystic fibrosis registries support disease modification by CFTR modulation with ivacaftor. Thorax. 2018. https://doi.org/10.1136/thoraxjnl-2017-210394.

13. Ferreira-Gonza'lez I, Marsal JR, Mitjavila F, Parada A, Ribera A, Cascant $\mathrm{P}$, et al. Patient Registries of Acute Coronary Syndrome: Assessing or Biasing the Clinical Real World Data? Circ Cardiovasc Qual Outcomes. 2009;2:540-7.

14. European Network of Centres for Pharmacoepidemiology and Pharmacovigilance [ENCePP]. Guide on methodological standards in pharmacoepidemiology: Patient Registries http://www. encepp.eu/standards_and_guidances/methodologicalGuide4 _3.shtml. Accessed 30 June 2019.

15. European Medicines Agency. Initiative for patient registries strategy and pilot phase. EMA/176050/2014 [Internet]. 2015. http://www.ema.europa.eu/docs/en_GB/document_library/Other /2015/10/WC500195576.pdf. Accessed 30 June 2019.

16. European Medicines Agency. Patient Registries Workshop, 28 October 2016-Observations and recommendations arising from the workshop. EMA/69716/2017 [Internet]. 2017. http://www. ema.europa.eu/docs/en_GB/document_library/Report/2017/02/ WC500221618.pdf. Accessed 30 June 2019.

17. European Medicines Agency. Report on Cystic Fibrosis registries: Workshop 14 June 2017, Patient Registries Initiative. https://www. ema.europa.eu/en/documents/report/report-cystic-fibrosis-regis tries_en.pdf. Accessed 30 June 2019.

18. European Medicines Agency. Report on Multiple Sclerosis registries: Workshop 7 July 2017, Patient Registries Initiative. https ://www.ema.europa.eu/en/documents/report/report-multiple-scler osis-registries_en.pdf. Accessed 30 June 2019.

19. European Medicines Agency. Report on CAR T-cell therapy registries: Workshop 9 February 2018, Patient Registries Initiative. https://www.ema.europa.eu/en/documents/report/report-car-t-celltherapy-registries-workshop_en.pdf. Accessed 30 June 2019.

20. European Medicines Agency. Report on Haemophilia registries: Workshop 8 June 2018, Patient Registries Initiative. https://www. ema.europa.eu/en/documents/report/report-haemophilia-registries -workshop_en.pdf. Accessed 30 June 2019.

21. Medical Dictionary for Regulatory Activities [MedDRA®]; https ://www.meddra.org/. Accessed 30 June 2019.
22. European Medicines Agency. Guideline on good pharmacovigilance practices; Module Vlll-Post- authorisation safety studies http://www.ema.europa.eu/docs/en_GB/document_library/Scien tific_guideline/2012/06/WC500129137.pdf. Accessed 30 June 2019.

23. European Medicines Agency. Qualification of novel methodologies for medicine development. http://www.ema.europa.eu/ema/ index.jsp?curl=pages/regulation/document_listing/document_listi ng_000319.jsp\&mid=WC0b01ac0580022bb0. Accessed 30 June 2019.

24. EU General Data Protection Regulation [GDPR]. https://eugdp rportal.godaddysites.com/. Accessed 30 June 2019.

25. Banzi R, Gerardi C, Bertele' V, Garattini S. Approvals of drugs with uncertain benefit-risk profiles in Europe. Eur J Intern Med. 2015;26(8):572-84.

26. Pease AM, Krumholz HM, Downing NS, Aminawung JA, Shah ND, Ross JS. Post-approval studies of drugs initially approved by the FDA on the basis of limited evidence: systematic review. BMJ. 2017;3(357):j1680.

27. Moore TJ, Furberg CD. Development times, clinical testing, postmarket follow-up, and safety risks for the new drugs approved by the US food and drug administration: the class of 2008. JAMA Intern Med. 2014;174(1):90-5.

28. European Medicines Agency. PRIME: PRIority MEdicines http:// www.ema.europa.eu/ema/index.jsp?curl=pages/regulation/gener al/general_content_000660.jsp\&mid=WC0b01ac05809f8439. Accessed 30 June 2019.

29. European Network of Centres for Pharmacoepidemiology and Pharmacovigilance [ENCePP]. ENCePP Resourced Database; http://www.encepp.eu/encepp/resourcesDatabase.jsp. Accessed 30 June 2019.

30. European Commission. European Reference Networks https:// ec.europa.eu/health/ern_en. Accessed 30 June 2019.

31. European Network for Health Technology Assessment [EUnetHTA] https://www.eunethta.eu/. Accessed 30 June 2019.

32. European Commission. Implementation report on the Commission Communication on rare diseases; 2014; https://ec.europa.eu/healt h/sites/health/files/rare_diseases/docs/2014_rarediseases_imple mentationreport_en.pdf. Accessed 30 June 2019.

33. European Commission. Horizon 2020 Work programme 20182020. http://ec.europa.eu/research/participants/data/ref/h2020/ wp/2018-2020/main/h2020-wp1820-health_en.pdf. Accessed 30 June 2019.

34. Healthcare Institute Netherlands. Register of registries https:// www.zorginstituutnederland.nl/actueel/nieuws/2019/04/02/zorgi nstituut-start-project-regie-op-registers-voor-dure-geneesmidd elen. Accessed 30 June 2019.

35. Swedish National Data Service. Register based research. https:// snd.gu.se/en/data-management/register-based-research. Accessed 30 June 2019.

36. European Commission. European Platform on Rare Diseases registration; Set of Common Data Elements. https://eu-rd-platform. jrc.ec.europa.eu/set-of-common-data-elements. Accessed 30 June 2019. 